RESEARCH ARTICLE

Ke Lou · Baotong Cui · Xiaojiao Zhang

\title{
Adaptive synchronization of two complex networks with delayed and non-delayed coupling
}

Received: 4 April 2011 / Accepted: 11 December 2011 / Published online: 21 April 2012

(C) The Author(s) 2012. This article is published with open access at Springerlink.com

\begin{abstract}
This paper studies the adaptive synchronization of two complex networks with non-delayed and delayed couplings, in which the coupling configuration matrices are not necessarily symmetric or irreducible. Considering the case of identical and nonidentical network topological structures, we obtain several criteria for synchronization of two complex networks based on the Lyapunov stability theory. Numerical simulations are presented to demonstrate the effectiveness of the proposed criteria.
\end{abstract}

\section{Mathematics Subject Classification 90B18}

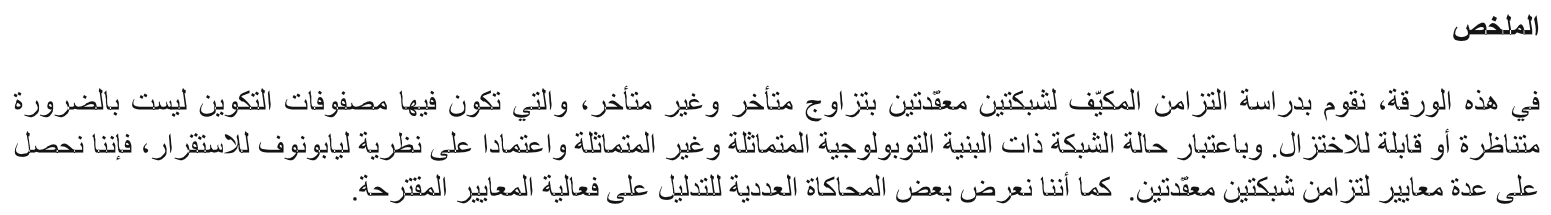

\section{Introduction}

The synchronization problem of complex networks has been one of the focus points in many research and application fields. In addition, time delays often appear in various complex dynamical networks due to the finite information transmission and processing speeds among the network nodes, and some of time delays cannot be ignored [18]. Therefore, much work has been done for the synchronization of complex networks with time delays in many literatures. Pecora and Carroll [9] studied the stability of synchronization in linearly coupled networks using the Master Stability function approach. Wang and Chen [12] investigated the synchronization problem for two specific kinds of complex networks such as small-world networks and scale-free networks,

K. Lou $(\bowtie)$

Key Laboratory of Advanced Process Control for Light Industry (Ministry of Education),

Jiangnan University, Wuxi 214122, China

E-mail: loukeauts@gmail.com

K. Lou

Anhui Province Key Laboratory of P.R. China (NO. 10111406006),

College of Electrical Engineering, Anhui Polytechnic University,

Wuhu, 241000 Anhui, China

B. Cui $\cdot$ X. Zhang

School of IoT Engineering, Jiangnan University, Wuxi 214122, China 
respectively. Zhang and Lu [19] introduced a complex networks model with delayed nodes, and obtained several novel criteria for globally exponentially asymptotic synchronization. Cai et al. [2] analyzed exponential synchronization of complex networks with nonidentical time-delayed nodes, and derived two effective control schemes. By utilizing geometrical decomposition of network states and linear matrix inequality method, Yang and Cao [17] derived several criteria for the exponential synchronization of complex networks with a coupling delay and impulses. Wang and Guan [13] investigated the chaos synchronization of a general complex network with coupling delays, and presented some delay-independent and delay-dependent criteria for exponential synchronization. In He et al. [5], generalized synchronization of two chaotic systems is investigated and some less restrictive criteria are obtained to guarantee the asymptotical stability of the error system between the response system and the auxiliary system, which indicates the drive-response systems are synchronized in a general sense. Cao et al. [3] analyzed the complete synchronization problem in an array of linearly stochastically coupled identical networks with time delays. Based on a simple adaptive feedback control scheme and some stochastic analysis techniques, several sufficient conditions are developed to guarantee the synchronization in an array of linearly stochastically coupled neural networks with time delays. Huang and Cao [6] introduced a novel coupling scheme with different coupling delays and investigated the generalized synchronization using the Lyapunov-Krasovskii functional method.

In the above references they all focused on the synchronization in one network that was named 'inner synchronization' because it was a collective behavior within a network. Different from the 'inner synchronization', Li et al. [7] firstly studied the synchronization between two complex networks which is called 'outer synchronization'. By designing effective adaptive controllers, Tang et al. [11] achieve synchronization between two complex networks with identical or nonidentical topological structures. In Sun et al. [10], a simple criterion for linear generalized synchronization between two complex networks with the same connection topologies is attained. Wang et al. [14] regard the outer synchronization between two delay-coupled complex networks with nonidentical topological structures and a noise perturbation. $\mathrm{Lu}$ and Cao [8] study the adaptive complete synchronization of chaotic and hyperchaotic systems with fully unknown parameters and derive an adaptive scheme to compensate for the effects of parameters' uncertainty based on the structure of chaotic systems. In Wu et al. [16], the problem of generalized outer synchronization between two completely different complex dynamical networks is investigated and a sufficient criterion for this generalized outer synchronization is derived based on Barbalat's lemma.

It is worth mentioning that these studies only consider the information transmission in complex network at time $t$ or at time $t-\tau$. However, in many circumstances, this simplification does not match satisfactorily the peculiarities of real networks: there exists the information communication of nodes not only at time $t$ but also at time $t-\tau$ [4]. In Guo et al. [4], the synchronization of the networks with both delayed and non-delayed coupling is introduced, and some sufficient conditions for the global synchronization by adding linear and adaptive feedback controllers to a part of nodes are obtained. For a given complex network with both delayed and non-delayed couplings, Wang et al. [15] proposed the minimum number of adaptive controllers under which synchronization can be achieved.

Motivated by above discussions, in this paper, we study the synchronization of two complex networks with both delayed and non-delayed coupling. In particular, we not only prove that the synchronization between two complex dynamical networks can be achieved by designing appropriate adaptive controller, but also consider the two complex networks with both delayed-coupled and non-delay coupled which can reflect a more realistic dynamical behavior of coupled networks in practice. For the coupling matrix, we do not assume that the coupling configuration matrix is symmetric or irreducible. Numerical examples are also provided to demonstrate the effectiveness of the theory.

\section{Complex networks model and preliminaries}

We consider a complex network consisting of $N$ identical nodes. Each node of the network is $n$-dimensional dynamical subsystem, which is described by

$$
\dot{x}_{i}(t)=f\left(x_{i}(t)\right)+\sum_{j=1}^{N} c_{i j} A x_{j}(t), \quad i=1,2, \ldots, N,
$$

where $x_{i}(t)=\left(x_{i 1}(t), \ldots, x_{i n}(t)\right)^{T} \in \mathbf{R}^{n}$ is the state vector of the $i$ th node, $f(\cdot) \in \mathbf{R}^{N}$ is a continuously differentiable vector function, and $\dot{x}_{i}(t)=f\left(x_{i}(t)\right)$ represents the dynamics of a single node. The matrix 
$A=\operatorname{diag}\left(a_{1}, a_{2}, \ldots, a_{n}\right) \in \mathbf{R}^{n \times n}$ is the inner connecting matrix of each node. The matrix $C=\left(c_{i j}\right)_{N \times N} \in$ $\mathbf{R}^{N \times N}$ is coupling configuration matrix describing the topological structure of the network, in which $c_{i j}>0$ if there is a link from node $j$ to node $i(i \neq j)$, and $c_{i j}=0$ otherwise; and the diagonal elements of $C$ are given by $c_{i i}=-\sum_{j=1, j \neq j}^{N} c_{i j}, i, j=1,2, \ldots, N$.

In [11], the network (1) was taken as the drive network, and the response network with an adaptive control scheme which is given by

$$
\dot{y}_{i}(t)=f\left(y_{i}(t)\right)+\sum_{j=1}^{N} d_{i j} A y_{j}(t)+u_{i}(t), \quad i=1,2, \ldots, N,
$$

where $y_{i}(t)=\left(y_{i 1}(t), \ldots, y_{i n}(t)\right)^{T} \in \mathbf{R}^{n}$ is the state vector of the $i$ th node in the response network, $D=$ $\left(d_{i j}\right)_{N \times N} \in \mathbf{R}^{N \times N}$ is the coupling configuration matrix, the $f$ and $A$ have the same meanings as those in (1), and $u_{i}(t)$ is the controller for node $i$ to be designed.

Based on the drive-response network systems (1)-(2), we will consider the following more general driveresponse networks with non-delayed and delayed coupling

$$
\begin{aligned}
& \dot{x}_{i}(t)=f\left(x_{i}(t)\right)+\sum_{j=1}^{N} c_{i j} A x_{j}(t)+\sum_{j=1}^{N} \widetilde{c}_{i j} \widetilde{A} x_{j}(t-\tau), \quad i=1,2, \ldots, N, \\
& \dot{y}_{i}(t)=f\left(y_{i}(t)\right)+\sum_{j=1}^{N} d_{i j} A y_{j}(t)+\sum_{j=1}^{N} \widetilde{d}_{i j} \widetilde{A} y_{j}(t-\tau)+u_{i}(t), \quad i=1,2, \ldots, N,
\end{aligned}
$$

where $x_{i}(t), y_{i}(t)$ and $f$ have the same meanings as those in (1)-(2), $\tau>0$ is the coupling delay. The coupling matrices $C=\left(c_{i j}\right)_{N \times N} \in \mathbf{R}^{N \times N}$ and $\widetilde{C}=\left(\widetilde{c}_{i j}\right)_{N \times N} \in \mathbf{R}^{N \times N}$ represent the topological structure for non-delayed configuration and delayed one, respectively. The $D$ and $\widetilde{D}$ have the same meanings as $C$ and $\widetilde{C}$ which are defined as in (1).

The interval conditions of the functional differential equations (3) and (4) are given by

$$
\begin{aligned}
& x_{i}(t)=\phi_{i}(t), \quad t \in[-\tau, 0], \quad i=1,2, \ldots, N, \\
& y_{i}(t)=\psi_{i}(t), \quad t \in[-\tau, 0], \quad i=1,2, \ldots, N .
\end{aligned}
$$

where $\phi_{i}(t)$ and $\psi_{i}(t)$ are continuous and differentiable vector-valued functions of $t \in[-\tau, 0]$.

Remark 2.1 In our complex network model, the coupling configuration matrices $C, \widetilde{C}, D$ and $\widetilde{D}$ don't need to be symmetric and irreducible. Moreover, it should be especially pointed out that our model has non-delayed and delayed couplings simultaneously. As a result, the model generalizes many realistic complex networks.

Definition 2.2 Let $x_{i}\left(t ; t_{0} ; \phi_{i}\right)$ and $y_{i}\left(t ; t_{0} ; \psi_{i}\right), i=1,2, \ldots N$ be the solutions of the delayed network (3) and (4), where $\phi_{i}=\phi_{i}(t) \in C\left([-\tau, 0], R^{n}\right)$ and $\psi_{i}=\psi_{i}(t) \in C\left([-\tau, 0], R^{n}\right)$ are initial conditions, $f: R \times \Omega \rightarrow R^{n}$ is continuously differentiable, $\Omega \subseteq R^{n}$. If there is a nonempty subset $\Lambda \subset \Omega$, such that $\phi_{i}$ takes values in $\Lambda$ and $x_{i}\left(t ; t_{0} ; \phi_{i}\right) \in \Omega$ for all $t \geq t_{0}, i=1,2, \ldots, N$, and

$$
\lim _{t \rightarrow \infty}\left\|y_{i}\left(t ; y_{0} ; \psi_{i}\right)-x\left(t ; x_{0} ; \phi_{i}\right)\right\|=0, \quad i=1,2, \ldots, N
$$

where $\|\cdot\|$ stands for the Euclidean vector norm, then the dynamical network (3) and (4) are said to be synchronized.

Throughout the paper, we make the following assumption and lemma.

Assumption 2.3 There exists a positive constant $L$ satisfying

$$
\|f(y)-f(x)\| \leq L\|y-x\| .
$$


Lemma 2.4 [1] For any vectors $x, y \in R^{n}$, and positive definite matrix $Q \in R^{n \times n}$, the following matrix inequality holds

$$
2 x^{T} y \leq x^{T} Q x+y^{T} Q^{-1} y .
$$

Define error vectors as

$$
e_{i}(t)=y_{i}(t)-x_{i}(t)
$$

where $i=1,2, \ldots, N$, then we have the error system as

$$
\begin{aligned}
\dot{e}_{i}(t)= & \dot{y}_{i}(t)-\dot{x}_{i}(t) \\
= & f\left(y_{i}(t)\right)-f\left(x_{i}(t)\right)+\sum_{j=1}^{N}\left(d_{i j} A y_{j}(t)-c_{i j} A x_{j}(t)\right) \\
& +\sum_{j=1}^{N}\left(\widetilde{d}_{i j} \widetilde{A} y_{j}(t-\tau)-\widetilde{c}_{i j} \widetilde{A} x_{j}(t-\tau)\right)+u_{i}(t),
\end{aligned}
$$

where $i=1,2, \ldots, N$. It is obvious that the stability of the synchronization between network (3) and network (4) is equivalent to stability of the zero solution of system (11).

\section{Synchronization criteria}

In this section, the synchronization of the drive-response networks with both delayed and non-delayed coupling is studied.

Theorem 3.1 Suppose that Assumption 2.3 holds. Under the condition of nonidentical configurations, i.e. $C \neq D$ and $\widetilde{C} \neq \widetilde{D}$, the drive network (3) and the response network (4) can realize synchronization, if the adaptive controller and control laws are designed as follows

$$
\begin{aligned}
u_{i}(t) & =\sum_{j=1}^{N} b_{i j} A y_{j}(t)+\sum_{j=1}^{N} \widetilde{b}_{i j} \widetilde{A} y_{j}(t-\tau)-g_{i} e_{i}(t), \\
\dot{b}_{i j} & =-e_{i}^{T}(t) A y_{j}(t), \dot{\widetilde{b}}_{i j}=-e_{i}^{T}(t) \widetilde{A} y_{j}(t-\tau), \\
\dot{g}_{i} & =\delta_{i}\left\|e_{i}(t)\right\|^{2}, \quad i=1,2, \ldots, N,
\end{aligned}
$$

where $\delta_{i}$ is positive constant.

Proof Construct the Lyapunov function candidate as follows

$$
\begin{aligned}
V(t)= & \frac{1}{2} \sum_{i=1}^{N} e_{i}^{T}(t) e_{i}(t)+\frac{1}{2} \sum_{i=1}^{N} \sum_{j=1}^{N}\left(d_{i j}+b_{i j}-c_{i j}\right)^{2}+\frac{1}{2} \sum_{i=1}^{N} \sum_{j=1}^{N}\left(\widetilde{d}_{i j}+\widetilde{b}_{i j}-\widetilde{c}_{i j}\right)^{2} \\
& +\frac{1}{2} \sum_{i=1}^{N} \frac{\left(g_{i}-l\right)^{2}}{\delta_{i}}+\sum_{i=1}^{N} \int_{t-\tau}^{t} e_{i}^{T}(\theta) e_{i}(\theta) d \theta,
\end{aligned}
$$

where $l$ is a positive constant to be determined. By using the inequality $x^{T} y \leq\left|x^{T} y\right| \leq\|x\|\|y\|$, along with Equations (11) and (12), we get

$$
\begin{aligned}
\dot{V}(t)= & \sum_{i=1}^{N} e_{i}^{T}(t) \dot{e}_{i}(t)+\sum_{i=1}^{N} \sum_{j=1}^{N}\left(d_{i j}+b_{i j}-c_{i j}\right) \dot{b}_{i j}+\sum_{i=1}^{N} \sum_{j=1}^{N}\left(\widetilde{d}_{i j}+\widetilde{b}_{i j}-\widetilde{c}_{i j}\right) \dot{\vec{b}}_{i j} \\
& +\sum_{i=1}^{N} \frac{1}{\delta_{i}}\left(g_{i}-l\right) \dot{g}_{i}+\sum_{i=1}^{N}\left(e_{i}^{T}(t) e_{i}(t)-e_{i}^{T}(t-\tau) e_{i}(t-\tau)\right)
\end{aligned}
$$




$$
\begin{aligned}
= & \sum_{i=1}^{N} e_{i}^{T}(t)\left[f\left(y_{i}(t)\right)-f\left(x_{i}(t)\right)+\sum_{j=1}^{N}\left(d_{i j} A y_{j}(t)-c_{i j} A x_{j}(t)\right)+\sum_{j=1}^{N}\left(\widetilde{d}_{i j} \widetilde{A} y_{j}(t-\tau)-\widetilde{c}_{i j} \widetilde{A} x_{j}(t-\tau)\right)\right. \\
& \left.+\sum_{j=1}^{N} b_{i j} A y_{j}(t)+\sum_{j=1}^{N} \widetilde{b}_{i j} \widetilde{A} y_{j}(t-\tau)-g_{i} e_{i}(t)+e_{i}(t)\right]-\sum_{i=1}^{N} \sum_{j=1}^{N}\left(d_{i j}+b_{i j}-c_{i j}\right) e_{i}^{T}(t) A y_{j}(t) \\
& -\sum_{i=1}^{N} \sum_{j=1}^{N}\left(\widetilde{d}_{i j}+\widetilde{b}_{i j}-\widetilde{c}_{i j}\right) e_{i}^{T}(t) \widetilde{A} y_{j}(t-\tau)+\sum_{i=1}^{N}\left(g_{i}-l\right)\left\|e_{i}(t)\right\|^{2}-\sum_{i=1}^{N} e_{i}^{T}(t-\tau) e_{i}(t-\tau) \\
= & \sum_{i=1}^{N} e_{i}^{T}(t)\left[f\left(y_{i}(t)\right)-f\left(x_{i}(t)\right)-g_{i} e_{i}(t)+e_{i}(t)\right]+\sum_{i=1}^{N} \sum_{j=1}^{N} e_{i}(t)^{T} c_{i j} A e_{j}(t) \\
& +\sum_{i=1}^{N} \sum_{j=1}^{N} e_{i}(t)^{T} \widetilde{c}_{i j} \widetilde{A} e_{j}(t-\tau)+\sum_{i=1}^{N}\left(g_{i}-l\right)\left\|e_{i}(t)\right\|^{2}-\sum_{i=1}^{N} e_{i}^{T}(t-\tau) e_{i}(t-\tau) \\
\leq & \sum_{i=1}^{N}\left[\left\|e_{i}(t)\right\|\left\|f\left(y_{i}(t)\right)-f\left(x_{i}(t)\right)\right\|+(1-l)\left\|e_{i}(t)\right\|^{2}\right]+\sum_{i=1}^{N} \sum_{j=1}^{N} e_{i}(t)^{T} c_{i j} A e_{j}(t) \\
& +\sum_{i=1}^{N} \sum_{j=1}^{N} e_{i}(t)^{T} \widetilde{c}_{i j} \widetilde{A} e_{j}(t-\tau)-\sum_{i=1}^{N}\left\|e_{i}(t-\tau)\right\|^{2} .
\end{aligned}
$$

By Assumption 2.3, we have

$$
\left\|f\left(y_{i}(t)\right)-f\left(x_{i}(t)\right)\right\| \leq L\left\|e_{i}(t)\right\| .
$$

By Lemma 2.4, we further have

$$
\begin{aligned}
\sum_{i=1}^{N} \sum_{j=1}^{N} \widetilde{c}_{i j} e_{i}^{T}(t) \widetilde{A} e_{j}(t-\tau) & \leq \frac{1}{2} \sum_{i=1}^{N} \sum_{j=1}^{N}\left[\widetilde{c}_{i j}^{2} e_{i}^{T}(t) Q e_{i}(t)+e_{j}^{T}(t-\tau) \widetilde{A}^{T} Q^{-1} \widetilde{A} e_{j}(t-\tau)\right] \\
& =\sum_{i=1}^{N} \sum_{j=1}^{N}\left[\frac{N}{4} \widetilde{c}_{i j}^{2} e_{i}^{T}(t) \widetilde{A}^{2} e_{i}(t)+\frac{1}{N} e_{j}^{T}(t-\tau) e_{j}(t-\tau)\right] \\
& \leq \frac{N}{4} \sum_{i=1}^{N} \sum_{j=1}^{N} \widetilde{c}_{i j}^{2}\left\|e_{i}^{T}(t)\right\|\left\|\widetilde{A}^{2}\right\|\left\|e_{i}(t)\right\|+\sum_{j=1}^{N}\left\|e_{j}(t-\tau)\right\|^{2} \\
& \leq \frac{N^{2}\left\|\widetilde{A}^{2}\right\| \max _{1 \leq i, j \leq N}\left\{\widetilde{c}_{i j}^{2}\right\}}{4} \sum_{i=1}^{N}\left\|e_{i}^{T}(t)\right\|^{2}+\sum_{j=1}^{N}\left\|e_{j}(t-\tau)\right\|^{2},
\end{aligned}
$$

where $Q=\frac{N}{2} \widetilde{A}^{2}>0$.

Substituting Equations (15) and (16) into Equation (14) yields

$$
\dot{V}(t) \leq\left(L+1-l+N\|A\| \max _{1 \leq i, j \leq N}\left\{\left|c_{i j}\right|\right\}+\frac{N^{2}\left\|\widetilde{A}^{2}\right\| \max _{1 \leq i, j \leq N}\left\{\widetilde{c}_{i j}^{2}\right\}}{4}\right) \sum_{i=1}^{N}\left\|e_{i}(t)\right\|^{2}
$$

Therefore, if $l$ is chosen as

$$
l>L+1+N\|A\| \max _{1 \leq i, j \leq N}\left\{\left|c_{i j}\right|\right\}+\frac{N^{2}\left\|\widetilde{A}^{2}\right\| \max _{1 \leq i, j \leq N}\left\{\widetilde{c}_{i j}^{2}\right\}}{4},
$$

we have $\dot{V}(t) \leq 0$. It is obvious that $\dot{V}(t)=0$ if and only if $e_{i}(t)=0$ for all $i=1,2, \ldots, N$. The orbits of (11) are globally asymptotically stable at $e_{i}(t)=0$. That is, the synchronization between the drive network (3) and the response network (4) can be realized under the adaptive controller (12). The proof is thus completed. 
Remark 3.2 The constant $\delta_{i}(i=1,2, \ldots, N)$ in the adaptive controller (12) can be chosen properly to adjust the speed of synchronization. A lager adaptive gain $\delta_{i}$ would lead to faster synchronization.

To make Theorem 3.1 more applicable, we give the following corollaries.

Corollary 3.3 Suppose that Assumption 2.3 holds. The drive network (3) and the response network (4) can realize synchronization, if the adaptive controller and control laws are designed as follows

$$
\begin{gathered}
u_{i}(t)=\sum_{j=1}^{N} b_{i j} A y_{j}(t)+\sum_{j=1}^{N} \widetilde{b}_{i j} \widetilde{A} y_{j}(t-\tau)-g_{i} e_{i}(t), \\
\dot{g}_{i}=\delta_{i}\left\|e_{i}(t)\right\|^{2}, \quad i=1,2, \ldots, N \\
\left\{\begin{array} { l l } 
{ \dot { \tilde { b } } _ { i j } = - e _ { i } ^ { T } ( t ) \widetilde { A } y _ { j } ( t - \tau ) , } & { \widetilde { c } _ { i j } \neq \widetilde { d } _ { i j } } \\
{ \dot { \tilde { b } } _ { i j } = 0 , } & { \widetilde { c } _ { i j } = \widetilde { d } _ { i j } }
\end{array} \left\{\begin{array}{ll}
\dot{b}_{i j}=-e_{i}^{T}(t) A y_{j}(t), & c_{i j} \neq d_{i j} \\
\dot{b}_{i j}=0, & c_{i j}=d_{i j}
\end{array}\right.\right.
\end{gathered}
$$

where $\delta_{i}$ is positive constant, $i=1,2, \ldots, N$.

Corollary 3.4 Suppose that Assumption 2.3 holds. The drive network (3) and the response network (4) have the identical configuration matrices, i.e., $C=D$ and $\widetilde{C}=\widetilde{D}$, then the two complex networks can realize synchronization using the following adaptive controller

$$
u_{i}(t)=-g_{i} e_{i}(t), \quad \dot{g}_{i}=\delta_{i}\left\|e_{i}(t)\right\|^{2}, \quad i=1,2, \ldots, N,
$$

where $\delta_{i}$ is positive constant.

The proofs of Corollaries 3.3 and 3.4 follow directly from Theorem 3.1 and are omitted here.

\section{Numerical simulations}

In this section, two numerical examples are used to show the effectiveness of the proposed synchronization criteria. We consider the chaotic Lorenz system as the node dynamic system of the complex network. A chaotic Lorenz system can be described by

$$
\begin{aligned}
\dot{x}_{i} & =f\left(x_{i}\right)=\left(\begin{array}{lll}
-a & a & 0 \\
c & -1 & 0 \\
0 & 0 & -b
\end{array}\right)\left(\begin{array}{l}
x_{i 1} \\
x_{i 2} \\
x_{i 3}
\end{array}\right)+\left(\begin{array}{l}
0 \\
-x_{i 1} x_{i 3} \\
x_{i 1} x_{i 2}
\end{array}\right) \\
& \triangleq H x_{i}+W\left(x_{i}\right)
\end{aligned}
$$

where $i=1,2, \ldots, 5, a=10, b=8 / 3$ and $c=28$, the system has a chaotic attractor. For any two state vectors $x_{i}$ and $x_{j}$, there exists a positive constant $r$ such that

$$
\left|W\left(x_{j}\right)-W\left(x_{i}\right)\right| \leq r\left|x_{j}-x_{i}\right|
$$

hence the Assumption 2.3 is satisfied (for details, see Ref. [11]).

Example 4.1 In the first example, both the drive complex network (3) and the response network (4) are assumed to have the same configuration matrices, i.e., $C=D$ and $\widetilde{C}=\widetilde{D}$, which are described by

$$
C=\left[\begin{array}{cccccc}
-2 & 1 & 1 & -2 & 1 & 1 \\
-1 & -3 & 0 & 1 & 2 & 1 \\
1 & 0 & -3 & 1 & 1 & 0 \\
1 & 1 & 1 & -4 & 1 & 0 \\
1 & 1 & 1 & 0 & -4 & 1 \\
1 & 1 & 0 & 0 & 0 & -2
\end{array}\right], \quad \widetilde{C}=\left[\begin{array}{cccccc}
-2 & 1 & 1 & 1 & -1 & 0 \\
-1 & -2 & 0 & 1 & 1 & 1 \\
1 & 0 & -3 & 1 & 1 & 0 \\
0 & 1 & 1 & -3 & 1 & 0 \\
1 & 1 & 1 & 0 & -4 & 1 \\
1 & 1 & 1 & 0 & 0 & -3
\end{array}\right]
$$

The inner connection matrices are taken as identity matrices of dimension 3, i.e. $A=\widetilde{A}=I_{3}$. It is assumed that the coupling delay is $\tau=0.5$. Then, according to Corollary 3.4, we derive the control scheme by Eq. (20).

The trajectories of synchronization errors $e_{i 1}(t), e_{i 2}(t)$ and $e_{i 3}(t)$ are shown in Fig 1. As described in Fig. 1, it can be observed that all of the state errors $e_{i 1}(t), e_{i 2}(t)$ and $e_{i 3}(t)$ tend to zero, which implies that two networks achieve synchronization. 

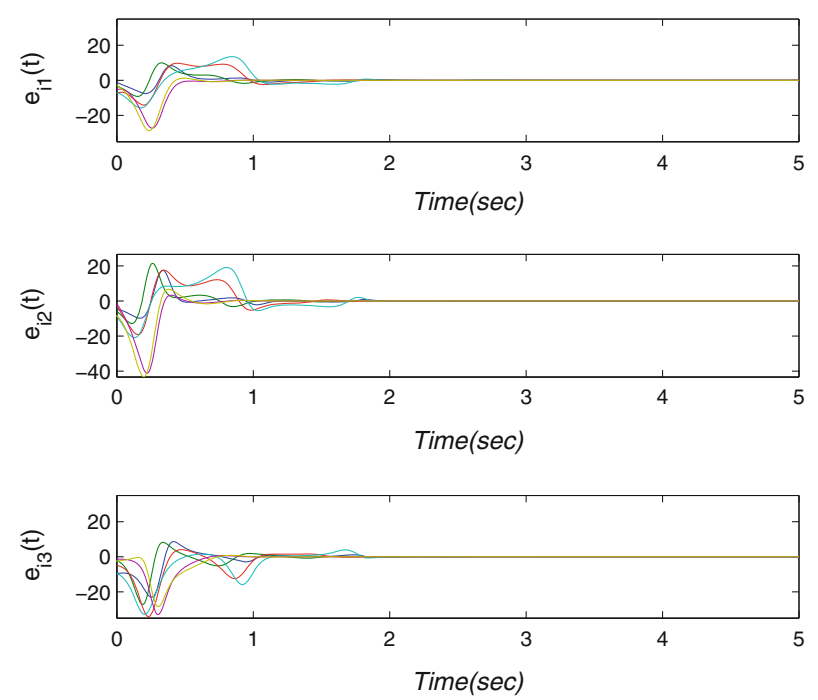

Fig. 1 Synchronization errors $e_{i 1}(t), e_{i 2}(t), e_{i 3}(t)(i=1,2, \ldots, 6)$ under updating law (20)

Example 4.2 The second example considers the case in which the drive complex network (3) and the response network (4) have different configuration matrices. Suppose that the configuration matrices for the drive complex network (3) $C$ and $\widetilde{C}$ as in the above subsection, and the configuration matrices for the response complex network (4) are given as follows

$$
D=\left[\begin{array}{llllll}
-1 & 0 & 1 & -1 & 0 & 1 \\
-1 & -2 & 0 & 0 & 3 & 0 \\
1 & 0 & -2 & 1 & 0 & 0 \\
1 & 0 & 1 & -3 & 1 & 0 \\
0 & 2 & 1 & 0 & -3 & 0 \\
1 & 1 & 0 & 1 & 0 & -3
\end{array}\right], \quad \widetilde{D}=\left[\begin{array}{llllll}
-3 & 0 & 0 & 1 & 2 & 0 \\
-2 & -2 & 0 & 3 & 1 & 0 \\
1 & 0 & -2 & 0 & 1 & 0 \\
0 & 1 & 1 & -3 & 1 & 0 \\
1 & 0 & 1 & 0 & -3 & 1 \\
1 & 1 & 1 & 0 & 0 & -3
\end{array}\right]
$$

We also assume that $A=\widetilde{A}=I_{3}$ and $\tau=0.5$. Then, by using the adaptive control algorithm (12) of Theorem 3.1. Figure 2 plots the trajectories of synchronization errors $e_{i 1}(t), e_{i 2}(t)$ and $e_{i 3}(t)$ between the two complex networks, which shows the realization of synchronization between the two complex networks.
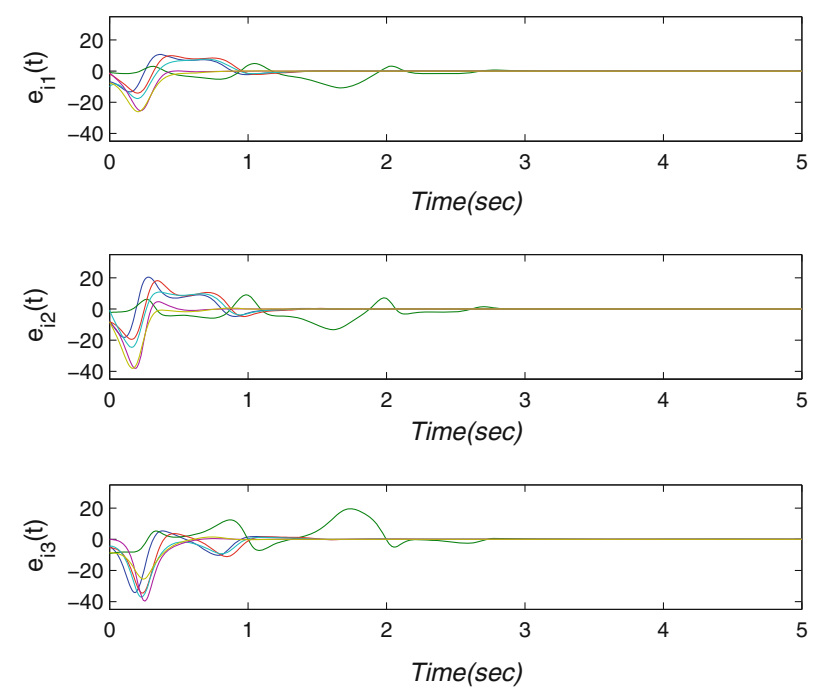

Fig. 2 Synchronization errors $e_{i 1}(t), e_{i 2}(t), e_{i 3}(t)(i=1,2, \ldots, 6)$ under updating law (12) 


\section{Conclusion}

In this paper, the adaptive synchronization problem for drive-response complex networks with non-delayed and delayed coupling has been studied. Considering the case of identical and nonidentical network topological structures, the adaptive controllers and update laws are designed to ensure synchronization between two complex networks. Additionally, two corollaries are also obtained. Moreover, the coupling configuration matrices are not necessarily symmetric or irreducible. Numerical simulations are provided to verify the effectiveness of the proposed scheme.

Acknowledgments This work was supported by National Natural Science Foundation of China (Nos. 61174021, 61104155).

Open Access This article is distributed under the terms of the Creative Commons Attribution License which permits any use, distribution, and reproduction in any medium, provided the original author(s) and the source are credited.

\section{References}

1. Boyd, S.; ElGhaoui, L.; Feron, E.; Balakrishnan, V.: Linear Matrix Inequalities in System and Control Theory. SIAM, Philadelphia (1994)

2. Cai, S.; He, Q.; Hao, J.; Liu, Z.: Exponential synchronization of complex networks with nonidentical time-delayed dynamical nodes. Phys. Lett. A 374, 2539-2550 (2010)

3. Cao, J.; Wang, Z.; Sun, Y.: Synchronization in an array of linearly stochastically coupled networks with time delays. Physica A 385, 718-728 (2007)

4. Guo, W.; Austin, F.; Chen, S.; Sun, W.: Pinning synchronization of the complex networks with non-delayed and delayed coupling. Physica A 373, 1565-1572 (2009)

5. He, W.; Cao, J.: Generalized synchronization of chaotic systems: the auxiliary system approach via matrix measure. Chaos 19(1), 013118-1-013118-10 (2009)

6. Huang, X.; Cao, J.: Generalized synchronization for delayed chaotic neural networks: a novel coupling scheme. Nonlinearity 19, 2797-2811 (2006)

7. Li, C.; Sun, W.; Kurths, J.: Synchronization between two coupled complex networks. Phys. Rev. E 76, 046204-1-046204-6 (2007)

8. Lu, J.; Cao, J.: Adaptive complete synchronization of two identical or different chaotic (hyperchaotic) systems with fully unknown parameters. Chaos 15(4), 043901-1-043901-10 (2005)

9. Pecora, L.M.; Carroll, T.L.: Master stability function for synchronized coupled systems. Phys. Rev. Lett. 80, 2109-2112 (1998)

10. Sun, M.; Zeng, C.; Tian, L.: Linear generalized synchronization between two complex networks. Commun. Nonlinear Sci. Numer. Simul. 15, 2162-2167 (2010)

11. Tang, H.; Chen, L.; Lu, J.; Tse, C.K.: Adaptive synchronization between two complex networks with nonidentical topological structures. Physica A 387, 5623-5630 (2008)

12. Wang, X.; Chen, G.: Synchronization in small-word dynamical networks. J. Bifurc. Chaos 12, 187-192 (2002)

13. Wang, B.; Guan, Z.: Chaos synchronization in general complex dynamical networks with coupling delays. Nonlinear Anal. Real World Appl. 11, 1925-1932 (2010)

14. Wang, G.; Cao, J.; Lu, J.: Outer synchronization between two nonidentical networks with circumstance noise. Physica A 389, 1480-1488 (2010)

15. Wang, Z.; Huang, L.; Zuo, Y.: Synchronization analysis of networks with both delayed and non-delayed couplings via adaptive pinning control method. Commun. Nonlinear Sci. Numer. Simul. 15, 4202-4208 (2010)

16. Wu, X.; Zheng, W.; Zhou, J.: Generalized outer synchronization between complex dynamical networks. Chaos 19, 013109$1-013109-9$ (2009)

17. Yang, Y.; Cao, J.: Exponential synchronization of the complex dynamical networks with a coupling delay and impulsive effects. Nonlinear Anal. Real World Appl. 11, 1650-1659 (2010)

18. Yue, D.; Li, H.: Synchronization stability of continuous/discrete complex dynamical networks with interval time-varying delays. Neurocomputing 73, 809-819 (2010)

19. Zhang, Q.; Lu, J.: Synchronization of a general delayed complex dynamical network via adaptive feedback. In: Proceedings of 2008 IEEE International Conference on Networking, Sensing and Control, Sanya, China, pp. 1818-1822 (2008) 